\title{
Safety and cost-effectiveness of outpatient thyroidectomy
}

\author{
$A$ retrospective observational study
}

Mohammed AlEssa, MBBS, FRCSC, Samiah S. Al-Angari, MBBS, MPH, Mohammed Jomah, MBBS, MSC, Aqeel AlOqaili, MBBS, SBORL, Muhammad Mujammami, MBBS, SF-Endo, Hadi A. Al-Hakami, MD, Saleh F. Al-Dhahri, MBBS, FRCSC.

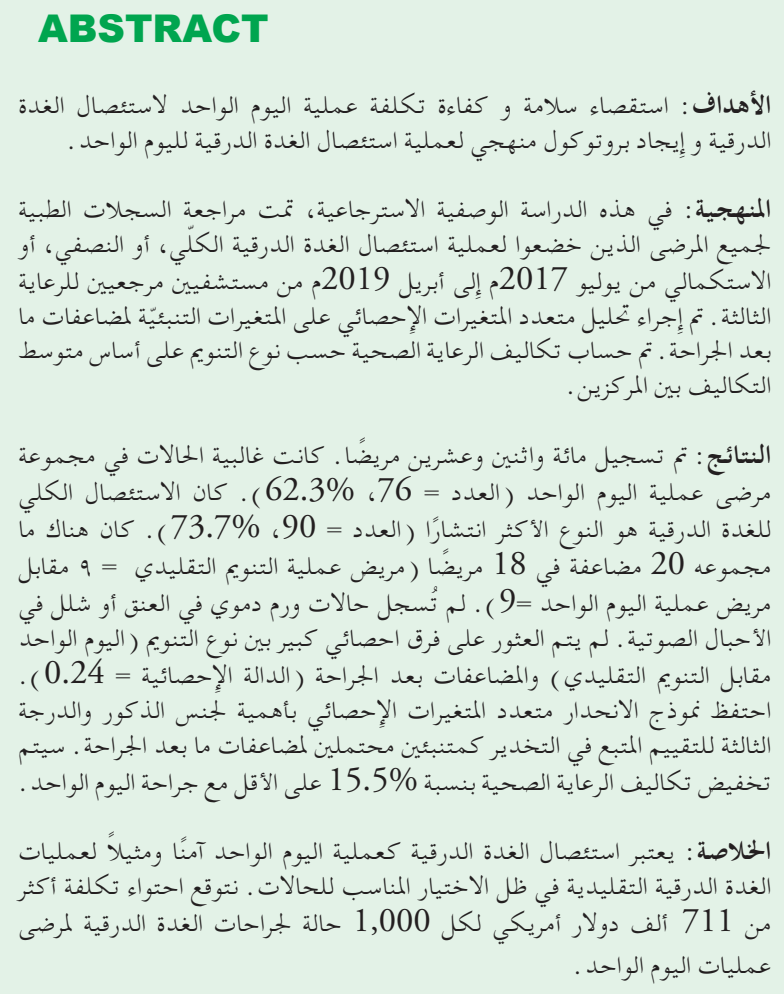

Objectives: To investigate the safety and cost-effectiveness of outpatient thyroidectomy and provide a systematic postoperative protocol for safe discharge.

Methods: In this retrospective review, the medical records of all patients who underwent total, hemi, or completion thyroidectomy from July 2017 to April 2019 at 2 tertiary care hospitals were reviewed. Multivariable analysis was performed on the potential predictors of postoperative complications. Healthcare costs were calculated by the type of admission based on the average costs at the 2 centers.

Results: One hundred twenty-two patients were enrolled in this study. The majority of cases were in the outpatient group $(n=76,62.3 \%)$. Total thyroidectomy was the most prevalent type of surgery $(n=90,73.7 \%)$. There were a total of 20 complications in 18 patients (inpatient $=9$ versus [vs.] outpatient=9). No cases of cervical hematoma or bilateral vocal cord paralysis were encountered. No significant difference was found between the type of admission (outpatient vs. inpatient) and postsurgical complications $(p=0.24)$. The multivariable regression model retained significance for male gender and American Society of Anesthesiologists Classification III as potential predictors of postoperative complications. Healthcare costs would be reduced by at least $15.5 \%$ with the implementation of outpatient surgery.

Conclusion: Outpatient thyroidectomy is as safe as inpatient thyroidectomy given the proper selection of cases. We project cost containment of over $\$ 711$ thousand per 1,000 cases for outpatient thyroid surgeries.

Keywords: thyroid, thyroidectomy, day surgery, procedure, ambulatory surgical, safety, outpatient surgery

Saudi Med J 2021; Vol. 42 (2): 188-195

doi: 10.15537/smj.2021.2.25686

From the Department of Otolaryngology - Head \& Neck Surgery (AlEssa, Al-Angari, Jomah, Al-Dhahri), College of Medicine; from the Division of Endocrinology and Metabolism, Department of Medicine (Mujammami), King Saud University; from the Department of Otolaryngology - Head \& Neck Surgery (AlEssa), King Abdulaziz Medical City, National Guard Health Affairs; from the Division of Otolaryngology - Head \& Neck Surgery, Department of Surgery (AlOqaili), King Abdullah bin Abdulaziz University Hospital; from the Department of Otolaryngology - Head \& Neck Surgery (Al-Dhahri), King Fahad Medical City, Riyadh; and from the Department of Otolaryngology - Head \& Neck Surgery (Al-Hakami), College of Medicine, King Saud bin Abdulaziz University for Health Sciences, Jeddah, Kingdom of Saudi Arabia.

Received 15th November 2020. Accepted 30th December 2020.

Address correspondence and reprint request to: Dr. Samiah S. Al-Angari, Department of Otolaryngology - Head \& Neck Surgery, College of Medicine, King Saud University, Riyadh, Kingdom of Saudi Arabia.

E-mail: samalangari@ksu.edu.sa

ORCID ID: https://orcid.org/0000-0002-8950-0524 
$\mathrm{T}$ hyroidectomy is currently one of the most commonly performed procedures nowadays, and is usually performed when malignancy is suspected or when the thyroid gland is enlarged, resulting in local compressive symptoms or cosmetic disfigurement. Similar to other surgical procedures, thyroidectomy carries potential risks and complications. Apart from anesthesia-related complications, the most common surgery-related intraoperative complications of thyroidectomy include recurrent laryngeal nerve injury, parathyroid gland injury, and excessive bleeding due to major vascular injury or poor hemostasis. Postoperatively, airway compromise due to vocal folds paralysis or hematoma collection, hypocalcemia, and surgical wound infection may be encountered. Identifying these complications in a timely manner can minimize further morbidity and prevent mortality. ${ }^{1-3}$ Therefore, careful surgical techniques combined with thorough postoperative evaluation must be undertaken to avoid dreadful consequences. ${ }^{3,4}$

A trending shift toward outpatient surgery has arisen in the past decade, as a growing body of evidence has advocated outpatient thyroid surgery and underscored differentiated selection criteria. ${ }^{1}$ This is especially apparent in the United States (U.S.), wherein outpatient procedures comprise over $70 \%$ of all elective surgical procedures. ${ }^{5}$ This change is partly driven economically by the benefit of healthcare cost containment, particularly in times at which costs and expenditures may influence healthcare access and quality of care. Additionally, short-stay procedures increase the availability of hospital beds and reduce the occurrence of nosocomial infections postoperatively. Sun et $\mathrm{al}^{6}$ reported that the overall total of outpatient thyroid surgeries has increased by $61 \%$, reaffirming the advantages perceived by surgeons in ambulatory settings.

Several institutions have implemented the practice of performing same-day surgeries and observing patients for a short period after surgery. ${ }^{1}$ Despite the opposing opinions on the feasibility of same-day thyroidectomy, numerous studies have demonstrated its safety, provided that cases are appropriately selected and provided that a methodical perioperative care protocol is charted to ensure the patient. ${ }^{1,2,7}$

Disclosure. Authors have no conflict of interests, and the work was not supported or funded by any drug company.
In 2013, the American Thyroid Association (ATA) published a statement on ambulatory thyroidectomy, providing a road map with a list of relative contraindications and eligibility criteria for such cases. ${ }^{1}$ Preoperative planning, patient education, and proper communication between team members have been proposed as prerequisites for safe surgery. Several recommendations regarding technical intraoperative and postoperative care have been suggested as well. ${ }^{1}$ The Association Francophone de Chirurgie Endocrinienne (AFCE) published recommendations based on the current practice of ambulatory thyroidectomy, which similarly stressed the importance of careful patient selection for same-day surgical procedures as well as adequate patient and family education. ${ }^{2}$

This study aim to assess the safety of outpatient thyroid surgery by measuring the rate of complications, the association of postsurgical complications with the type of admission (outpatient versus [vs.] inpatient), the readmission rate of ambulatory thyroid surgery, and the effectiveness of empirical oral calcium as a prophylaxis in such cases. We also reflect on healthcare costs by admission type.

Methods. This retrospective study reviewed the collected data of all patients who underwent total, hemi, or completion thyroidectomy by a single faculty surgeon from July 2017 to April 2019 at King Abdulaziz University Hospital and King Abdulaziz Medical City, Riyadh, Saudi Arabia. This study was conducted in accordance with the Declaration of Helsinki, as revised in 2013, with an Institutional Review Board approval from King Abdulaziz Medical City (IRB \#19/155/R). The routine preoperative assessment of each patient included the collection of the patient's full medical history, a physical examination, a thyroid ultrasound, fine needle aspiration (FNA), vocal folds function assessment using flexible laryngoscopy, and laboratory tests (complete blood count; coagulation profile; renal function; thyroid function test, thyroid stimulating hormone and free T4; parathyroid hormone [PTH]; vitamin $\mathrm{D}$; and corrected calcium).

Inclusion criteria included patients who underwent thyroid surgery and were not restricted by diagnosis (benign vs. malignant), type of surgery (total vs. hemi vs. completion thyroidectomy), age, or gender. We excluded any patient with American Society of Anesthesiology Classification (ASA) IV status. However, the selection criteria for outpatient surgery were applied by the first author (M.A.) according to a pre-defined algorithm (Figure 1). We defined the inpatient group as patients who were kept for overnight observation, 


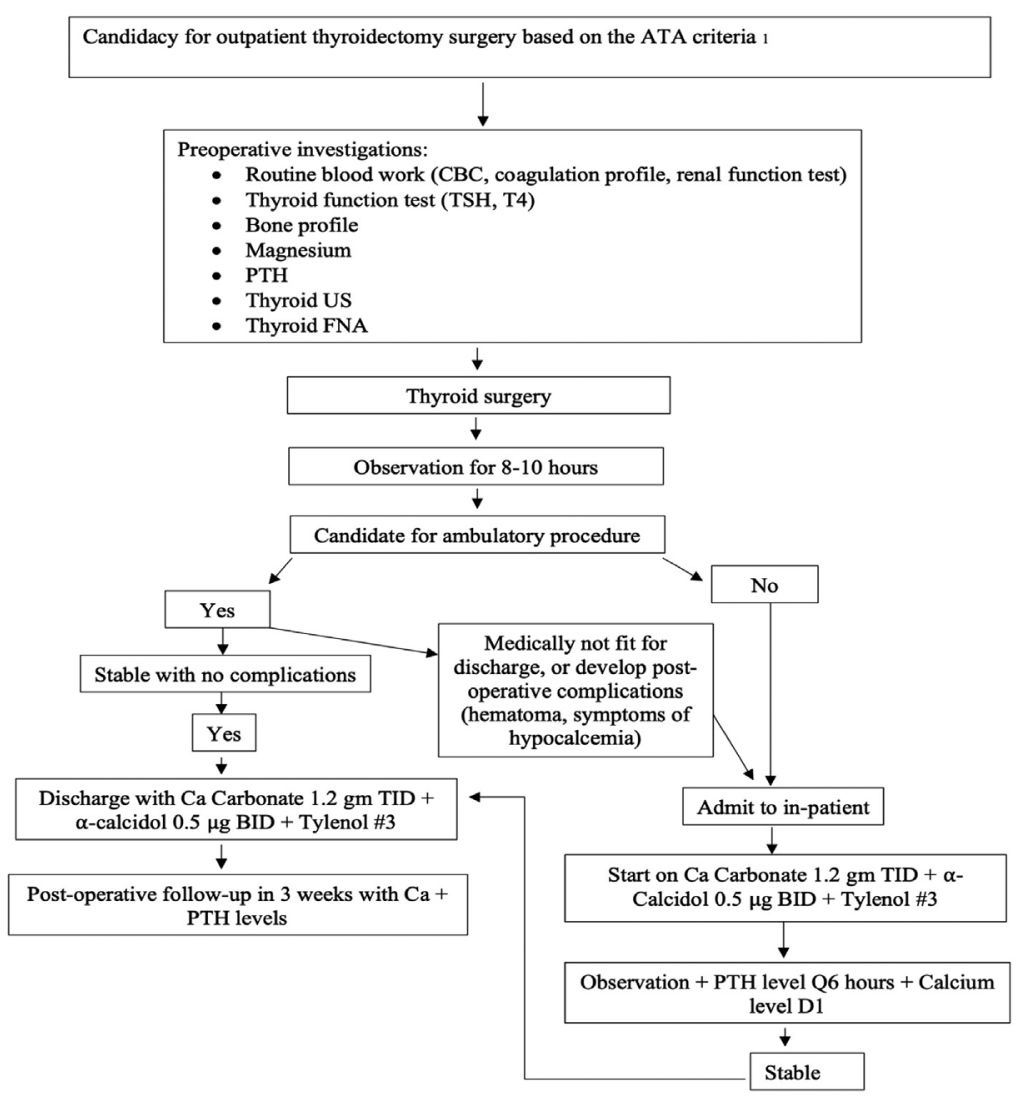

Figure 1 - Algorithm of outpatient total thyroidectomy. FNA: fine needle aspiration, PTH: parathyroid hormone, US: ultrasound, CBC: complete blood count, TSH: thyroid stimulating hormone, TID; trice a day, BID: twice a day

while the outpatient group was defined as patients who were observed for one hour in the recovery room and for $8-10$ hours afterward in the general ward prior to discharge.

Allsurgerieswere performed byasinglefacultysurgeon (M.A.) in 2 tertiary care centers with the assistance of an in-training (resident or fellow) surgeon. The single faculty surgeon (M.A.) performing these procedures had 7 years of surgical experience, with an average case load of 260 cases/year and an overall complication rate of $0.09 \%$ pertaining to unilateral temporary vocal cord paralysis. All thyroidectomy procedures were performed in accordance with a standardized technique. A Kocher incision was utilized and followed by strap muscles dissection. In all cases, the recurrent laryngeal nerve and at least 3 parathyroid glands with good blood supply were identified. A disposable nerve stimulator was used at the end of each procedure to confirm the integrity of the recurrent laryngeal nerve on each side. Hemostasis was maintained throughout the surgical procedure and confirmed at the end of the procedure prior to wound closure by careful inspection of the thyroid gland bed with the Valsalva maneuver $(40 \mathrm{mmHg}$ of pressure for 20 seconds) to search for any sources of bleeding.

All data were collected on an Excel spreadsheet (Microsoft ${ }^{\oplus}$ Excel for Mac V16.19, 2018) that included basic demographic data, type of admission (outpatient vs. inpatient), ASA classification, type of surgery, pathological diagnosis (benign vs. malignant), postoperative complications, type of complications, readmission, emergency room (ER) visits, postoperative calcium (low if corrected calcium $<2.2 \mathrm{mmol} / \mathrm{l}$ ), and PTH (low if PTH $<1.6 \mathrm{pg} / \mathrm{ml}$ ) levels. For the outpatient group, the first measurements of calcium and PTH levels were taken 3 weeks postoperatively, corresponding to the first postoperative clinic visit; for the inpatient group, these measurements were taken on day one postoperatively, followed by a second set of measurements 3 weeks postoperatively. Immediately after the operation, all patients in both groups were 
started on prophylactic calcium supplements $(1,200 \mathrm{mg}$ of calcium carbonate taken orally 3 times daily in the first week, tapered to twice daily in the 2 nd week, and once daily in the 3 rd week) in addition to $\alpha$-calcidol $(0.5 \mu \mathrm{g}$ twice daily for 3 weeks). Prior to discharge, all patients were assessed by the surgeon for any symptoms or signs of postoperative complications pertaining to hematoma collection, hypocalcemia, or voice change and stridor.

The cost of the surgical procedure and hospital stay were obtained from the hospital registries of 2 governmental institutions, and the average was taken for the assessment of the surgical costs of both groups.

Statistical analysis. Descriptive analysis was interpreted using means (standard deviations [SD]) or counts with percentages. Group comparisons of the categorical variables were carried out using Chi-square or Fisher's exact tests where suitable. Mean differences in the continuous variables were tested using Student's t-test. We also performed a multivariable logistic regression of the potential predictors of postoperative complications. The following variables were introduced as independent categorical variables in the logistic regression model: age, gender, type of admission, and ASA classification. Age was transformed into a 3-level balanced categorical variable. We opted to include predictors based on their relevancy in clinical practice and selected the best fit model based on the lowest Akaike information criterion). Odds ratios were calculated with 95\% confidence intervals (CIs). All

Table 1 - Demographic and clinical characteristics of the $(n=122)$ patients enrolled in the study stratified by type of admission.

\begin{tabular}{|c|c|c|c|c|}
\hline Characteristic & $\begin{array}{l}\text { All cases } \\
(\mathrm{n}=122)\end{array}$ & $\begin{array}{l}\text { Outpatient } \\
(\mathrm{n}=76)\end{array}$ & $\underset{(n=46)}{\text { Inpatient }}$ & $P$-value \\
\hline Age (years) & $46(13.7)$ & $44(12.8)$ & $49.8(14.5)$ & $0.02^{* \dagger}$ \\
\hline $17-40$ & $45(35.4)$ & & & \\
\hline $41-53$ & $43(33.9)$ & & & \\
\hline $54-80$ & $39(30.7)$ & & & \\
\hline \multicolumn{5}{|l|}{ Gender } \\
\hline Female & $98(80.0)$ & $60(79.0)$ & $38(82.6)$ & \multirow{2}{*}{$0.62^{\ddagger}$} \\
\hline Male & $24(19.7)$ & $16(21.1)$ & $8(17.4)$ & \\
\hline \multicolumn{5}{|l|}{ Type of pathology } \\
\hline Benign & $71(58.2)$ & $44(57.9)$ & $27(58.7)$ & \multirow{2}{*}{$0.93^{\ddagger}$} \\
\hline Malignant & $51(41.8)$ & $32(42.1)$ & $19(41.3)$ & \\
\hline \multicolumn{5}{|l|}{ Type of surgery } \\
\hline Total thyroidectomy & $90(73.7)$ & $50(65.8)$ & $40(87.0)$ & \multirow{3}{*}{$0.029^{*}$} \\
\hline Hemithyroidectomy & $20(16.4)$ & $17(22.4)$ & $3 \quad(6.5)$ & \\
\hline Completion thyroidectomy & $12(9.8)$ & $9(11.8)$ & $3 \quad(6.5)$ & \\
\hline \multicolumn{5}{|l|}{ Complications } \\
\hline Yes & $18(14.8)$ & $9(11.9)$ & $9(19.6)$ & \multirow{2}{*}{$0.24^{\ddagger}$} \\
\hline No & $104(85.3)$ & $67(88.2)$ & $37(80.4)$ & \\
\hline \multicolumn{5}{|l|}{ ER visits and readmission } \\
\hline Yes & $8 \quad(6.6)$ & $6(75)$ & $2(25.0)$ & \multirow{2}{*}{$0.70^{\S}$} \\
\hline No & $114(93.4)$ & $70(61.4)$ & $44(38.6)$ & \\
\hline \multicolumn{5}{|c|}{$\begin{array}{l}\text { American Society of Anesthesiology } \\
\text { Classification }\end{array}$} \\
\hline I & $33(27.0)$ & $17(22.4)$ & $16(34.8)$ & \\
\hline II & $63(51.6)$ & $46(60.5)$ & $17(37.0)$ & \\
\hline III & $26(21.3)$ & $13(17.1)$ & $13(28.3)$ & \\
\hline Post-operative calcium $(\mathrm{n}=90)$ & $2.18 \quad(0.4)$ & $2.15 \quad(0.4)$ & $2.22(0.30)$ & $0.4^{\dagger}$ \\
\hline Post-operative PTH (n=84) & $3.08 \quad(2.3)$ & $3.37 \quad(2.5)$ & $2.73(1.9)$ & $0.19^{\dagger}$ \\
\hline
\end{tabular}

Values are presented as numbers and percentages (\%) or mean (standard deviation). ${ }^{*} p<0.05$, ${ }^{\dagger}$ Student's t-test, ${ }^{\ddagger}$ Chi-square test, ${ }^{\circledR}$ Fisher's exact test, PTH: parathyroid hormone 
tests were 2 -tailed and $p$-values $<0.05$ were considered significant. All statistical analyses were performed using SAS 9.4 software (SAS Institute Inc., Cary, NC, USA).

Results. One hundred and twenty-two patients were enrolled in the present study. There were $(n=76$, $62.3 \%)$ patients in the outpatient group and $(n=46$, $37.7 \%)$ in the inpatient group. The present sample had an overall mean age of 46 (13.7) years, benign pathology in approximately two-thirds of the sample, and a majority of female patients $(n=98,80 \%)$. The 2 groups (outpatient vs. inpatient) were homogenous in terms of gender, complications, and type of pathology but different in terms of age and type of surgery. The basic clinical characteristics and associations of the variables are presented in Table 1.

A total of 20 complicationswerefound postoperatively in 18 patients, equally distributed between the 2 groups. Hypocalcemia was the most common complication, followed by temporary unilateral vocal cord paralysis (Table 2). No cases of cervical hematoma or bilateral vocal cord paralysis were encountered. Of the 3 main complications (hypocalcemia, cervical hematoma, and vocal cord paralysis), a total of 8 patients $(6.5 \%)$ visited the ER; 7 of these patients presented with hypocalcemia symptoms (outpatient $\mathrm{n}=5$ vs. inpatient $\mathrm{n}=2$ ), and only one was readmitted due to delayed surgical emphysema after an outpatient total thyroidectomy (one month after the surgery). This could be attributed to the fact that the latter patient had nasopharyngeal carcinoma and underwent radiation therapy 6 months prior to surgery. Radiation therapy is a known risk factor for wound dehiscence.

All patients were seen in the follow-up clinic 3 weeks postoperatively. Calcium levels were available for 90 patients (mean $=2.18, \mathrm{SD}=0.37$, range: $0.78-2.99$ $\mathrm{mmol} / \mathrm{l}$ ), and PTH levels were available for 84 patients (mean=3.08, SD=2.26, range: $0.318-11.3 \mathrm{pg} / \mathrm{ml}$ ). There were no significant differences in calcium or PTH levels between the 2 groups (Table 1 ).

The bivariate analysis showed that there were no significant differences between postsurgical complications and type of admission (outpatient vs. inpatient) (OR=0.55, 95\% CI: 0.20-1.5, $p=0.24)$,

Table 2 - Surgical outcomes of the $(\mathrm{n}=18)$ patients with 20 complications, by admission type.

\begin{tabular}{lccc}
\hline Outcome & Outpatient & Inpatient & Total \\
\hline Hypocalcemia & $5(55.6)$ & $8(72.7)$ & $13(65.0)$ \\
Unilateral vocal cord paralysis & $3(33.3)$ & $3(27.3)$ & $6(30.0)$ \\
Delayed surgical emphysema & $1(11.1)$ & $0(0)$ & $1 \quad(5.0)$ \\
Total complications & $9(100)$ & $11(100)$ & $20(100)$ \\
\hline
\end{tabular}

Values are presented as numbers and percentages (\%).

Table 2 - Results of logistic regression with complications as the outcome in the $(\mathrm{n}=122)$ patients enrolled in the study.

\begin{tabular}{lccc}
\hline Variable & $\mathrm{OR}^{*}[95 \% \mathrm{CI}]^{\dagger}$ & $\chi^{2}$ & $P$-value \\
\hline Age (54-80, reference) & $4.7[0.986-22.43]$ & 3.77 & 0.052 \\
$\quad$ Age 17-40 & $3.295[0.706-15.37]$ & 2.3 & 0.12 \\
Age 41-53 & $4.36[1.18-16.127]$ & 4.87 & $0.027^{\ddagger}$ \\
Gender (Female, reference) & & & \\
$\quad$ Male & $0.35[0.11-1.11]$ & 3.15 & 0.075 \\
Type of admission (inpatient, reference) & & & \\
$\quad$ Outpatient & $3.377[0.676-16.855]$ & 2.2 & 0.13 \\
ASA classification (ASA I, reference) & $8.4[1.32-53.3]$ & 5.09 & $0.024^{\ddagger}$ \\
ASA II & & & \\
ASA III &
\end{tabular}

OR: *Odds ratio, 'Wald's confidence interval, ${ }^{\ddagger} p<0.05$, reference: reference group 
genders $(p=0.62)$, or types of pathology $(p=0.93)$. Furthermore, hospital visits to the ER and cases warranting postsurgical readmission were not associated with the type of admission ( $p=0.70)$. Only age $(p=0.02)$ and type of surgery $(p=0.029)$ were significant.

The adjusted multivariable logistic model showed that only male gender and ASA III retained significance, while the type of admission, age, female gender, and ASA I and II were not influential (Table 3).

Healthcare costs. The total cost of the thyroidectomy procedure across both institutions averaged Saudi Riyals (SAR) 14,517 (\$3,871), which equals the cost of an outpatient thyroidectomy procedure. With the inclusion of laboratory investigations, nursing care, and overnight stay incurred by inpatient admission, this cost rose to SAR17,184 $(\$ 4,582)$. There was a minimum difference of SAR2,667 (\$711.2) between inpatient and outpatient procedures, which corresponds to a $15.5 \%$ reduction in health care costs when the procedure is performed as an outpatient procedure.

Discussion. In the present study, both groups (outpatient vs. inpatient) were homogenous in terms of gender, complications, and type of pathology, but different in terms of age and type of surgery. The type of admission was not significantly associated with complications even after adjustment in the multivariable regression model. This inference is reassuring and positively builds upon previous literature advocating outpatient surgeries. The multivariable logistic model implied that males were more likely to develop complications than females and that those with ASA III were more likely to develop complications than those with ASA I.

There appears to be a pattern in the literature showing that a higher complications rate is associated with overnight stays compared to same-day surgeries. ${ }^{8-10}$ According to the American College of Surgeons National Surgical Quality Improvement Program registry, outpatient thyroidectomy surgeries had decreased odds of 30-day hospital readmission, which persisted even after the adjustment of preoperative and postoperative variables. The inference in these papers in general may not merely reflect a higher rate of complications in the inpatient groups in itself, as it is probably subjected to an expected selection bias (namely, probable allocation of high-risk patients as inpatients), but it may serve as a proxy for determining that outpatient thyroid surgeries carry at least a non-inferiority margin of safety that cannot be overlooked, especially with proper profiling of eligible cases in the hands of experienced surgeons. Moreover, it indirectly indicates the importance of adherence to a pre-defined eligibility criteria of individuals for outpatient procedures.

The most common complication encountered in the present sample was hypocalcemia, which was mostly transient. Thirteen patients (10.6\%) presented with symptomatic hypocalcemia despite the use of a prophylactic regimen. This percentage, however, is less than the prevalence rates published in the literature and in a previous report published by our group for temporary hypocalcemia following thyroidectomy.,11 The lower prevalence of hypocalcemia in the present study could be attributed to the use of prophylactic supplements. The use of a prophylactic postoperative calcium supplement \pm vitamin $\mathrm{D}(1,25$ dihydroxyvitamin $\mathrm{D})$ has been shown to decrease the incidence of hypocalcemia, and many institutions have adopted this practice with good outcomes. ${ }^{12-16}$

Previously, we extrapolated/correlated future calcium levels from PTH levels in the first hour following surgery. ${ }^{11}$ In this paper, we draw on the work of Dr. David Terris ${ }^{8,16}$, aiming once again to identify the percentage of patients in our cohort who would develop hypocalcemia with the inclusion of calcium and vitamin D supplements when included as a routine regimen.

Two U.S. national studies utilized national databases to examine the costs and complications of thyroidectomy. ${ }^{9,17}$ The average cost of an outpatient thyroidectomy was $\$ 5,617$, whereas the average cost of a thyroidectomy with overnight observation was $\$ 6,101$. Provided that healthcare costs in the U.S. are among the most expensive in the world, we observed an alignment in conclusions despite cost differences between the 2 countries (namely, U.S. and Saudi Arabia). Furthermore, Terris et $\mathrm{al}^{16}$ estimated a statistically significant difference of over $\$ 2,000$ between inpatient and outpatient surgeries, while the AFCE reported a $15-30 \%$ cost reduction for institutions. ${ }^{2}$

The current healthcare system in Saudi Arabia is mainly reliant on government coverage and partly reliant on employee-based private insurance. The governmental budget for the Ministry of Health $(\mathrm{MoH})$ in the fiscal year of 2020 accounted for $7.4 \%$ of the total gross domestic products, with a total budget exceeding SAR75 billion (over $\$ 20$ billion). ${ }^{18}$ As per the statistics conducted by the General Directorate of Statistics and Information in the $\mathrm{MoH}$ for the year of 2017, the individual spending per capita was $\$ 20,968$, with over 1.1 million surgical operations $(49,655$ surgeries in the otolaryngology specialty alone), 22.4 beds per 10,000 population, 3,272,561 inpatients, and 341 million lab investigations in all health sectors (governmental and private). Furthermore, the total number of beds for 
the otolaryngology specialty in governmental hospitals alone was 549 beds. ${ }^{19}$ This low bed availability for Otolaryngology - Head and Neck Surgery makes it more challenging for surgeons to accommodate and manage high numbers of cases in an inpatient setting.

However, this system is on the brink of changing into a predominantly privatized healthcare system funded and supervised by the government through a National Healthcare Insurance plan with the integration of the private sector and its subcontractors. Accordingly, the funding and appropriation scheme for the $\mathrm{MoH}$ will change with different levels of capitations. ${ }^{20} \mathrm{~A}$ privatized healthcare market implies the dominance of health insurance plans and out-of-pocket deductibles. In such systems, cost containment is crucial for all players (buyers, providers, and intermediaries).

Study limitations. The retrospective nature of this study renders selection bias a potential limitation which was reflected by younger age and less total thyroidectomy rate in the outpatient thyroidectomy group. Furthermore, the temporal difference in measuring PTH and calcium levels between the 2 groups (inpatient vs. outpatient) may have detected more hypocalcemia cases in the inpatient group than in the outpatient group.

In conclusion, we project that shifting thyroidectomy surgeries from inpatient to outpatient procedures will result in a $15.5 \%$ cost reduction. In institutions making this shift, we expect that cost containment will reach approximately SAR2,667,000 $(\$ 711,200)$ per 1,000 cases.

Acknowledgment. The authors gratefully acknowledge Scribendi (www.scribendi.com) for English language editing.

\section{References}

1. Terris DJ, Snyder S, Carneiro-Pla D, Inabnet WB III, Kandil E, Orloff L, et al. American Thyroid Association statement on outpatient thyroidectomy. Thyroid 2013; 23: 1193-1202.

2. Menegaux F, AFCE. Ambulatory thyroidectomy: recommendations from the Association Francophone de Chirurgie Endocrinienne (AFCE). Investigating current practices. J Visc Surg 2013; 150: 165-171.

3. Balentine CJ, Sippel RS. Outpatient thyroidectomy: is it safe? Surg Oncol Clin N Am 2016; 25: 61-75.

4. Dionigi G, Rovera F, Carrafiello G, Bacuzzi A, Boni L, Dionigi R. New technologies in ambulatory thyroid surgery. Int J Surg 2008; 6 Suppl 1: S22-S25.
5. Mirnezami R, Sahai A, Symes A, Jeddy T. Day-case and short-stay surgery: the future for thyroidectomy? Int J Clin Pract 2007; 61: 1216-1222.

6. Sun GH, DeMonner S, Davis MM. Epidemiological and economic trends in inpatient and outpatient thyroidectomy in the United States, 1996-2006. Thyroid 2013; 23: 727-733.

7. Parameswaran R, Sutaria R, Abdel-Aziz T, Sadler G, Mihai R. Changing trends in thyroid and parathyroid surgery over the decade: is same day discharge feasible in the United Kingdom? World J Surg 2015; 39: 2104.

8. Seybt MW, Terris DJ. Outpatient thyroidectomy: experience in over 200 patients. Laryngoscope 2010; 120: 959-963.

9. Khavanin N, Mlodinow A, Kim JY, Ver Halen JP, Antony AK, Samant S. Assessing safety and outcomes in outpatient versus inpatient thyroidectomy using the NSQIP: a propensity score matched analysis of 16,370 patients. Ann Surg Oncol 2015; 22: 429-436.

10. Khadra H, Mohamed S, Hauch A, Carter J, Hu T, Kandil E. Safety of same-day thyroidectomy: meta-analysis and systematic review. Gland Surg 2017; 6: 292-301.

11. Al-Dhahri SF, Mubasher M, Al-Muhawas F, Alessa M, Terkawi RS, Terkawi AS. Early prediction of oral calcium and vitamin $\mathrm{D}$ requirements in post-thyroidectomy hypocalcaemia. Otolaryngol Head Neck Surg 2014; 151: 407-414.

12. Antakia R, Edafe O, Uttley L, Balasubramanian SP. Effectiveness of preventative and other surgical measures on hypocalcemia following bilateral thyroid surgery: a systematic review and meta-analysis. Thyroid 2015; 25: 95-106.

13. Segel JM, Duke WS, White JR, Waller JL, Terris DJ. Outpatient thyroid surgery: Safety of an optimized protocol in more than 1,000 patients. Surgery 2016; 159: 518-523.

14. Nelson KL, Hinson AM, Lawson BR, Middleton D, Bodenner DL, Stack BC Jr. Postoperative calcium management in same-day discharge thyroid and parathyroid surgery. Otolaryngol Head Neck Surg 2016; 154: 854-860.

15. Perera AH, Patel SD, Law NW. Thyroid surgery as a 23-hour stay procedure. Ann R Coll Surg Engl 2014; 96: 284-288.

16. Terris DJ, Moister B, Seybt MW, Gourin CG, Chin E. Outpatient thyroid surgery is safe and desirable. Otolaryngol Head Neck Surg 2007; 136: 556-559.

17. Marino M, Spencer H, Hohmann S, Bodenner D, Stack BC Jr. Costs of outpatient thyroid surgery from the University Health System Consortium (UHC) database. Otolaryngol Head Neck Surg 2014; 150: 762-769.

18. Saudi Arabian Ministry of Health. Budget of Saudi Ministry of Health 2017 (1438). [Updated 2020. Accessed 2020 December 26]. Available from URL: https://www.moh.gov.sa/ en/Ministry/About/Pages/Budget.aspx

19. Saudi Arabian Ministry of Health. Annual Statistical Book. General Directorate of Statistics and Information. [Updated 2018. Accessed 2019 July 17]. Available from URL: https:// www.moh.gov.sa/en/Ministry/Statistics/book/Pages/default. aspx

20. Saudi Arabian Ministry of Health. Health Sector Transformation Strategy. [Updated 2019. Accessed 2020 November 1]. Available from URL: https://www.moh.gov.sa/Ministry/About/ Documents/2019-004.pdf 